\title{
Research on Knowledge Modeling for Bonded Repair of Composite Aircraft Component
}

\author{
Qiang $\mathrm{He}^{*}$, Wenfeng Yang and Qingru Tang \\ Civil Aviation Flight University of China, Guanghan 618307, China \\ ${ }^{*}$ Corresponding author
}

\begin{abstract}
Advanced composite materials have gained popularity in aircraft industry. The widespread application leads to the requirement of high efficient and reliable maintenance technology for composite components. Bonded composite repairs are preferred as they provide enhanced stress transfer mechanisms, joint efficiencies and aerodynamic performance. Because of the inherent complex damage behavior, the bonded repairs rely on the maintenance knowledge and experience heavily. The maintenance knowledge of composite components can improve the maintenance quality and efficiency. To accumulate and apply the knowledge, bonded repair knowledge modeling method based on ontology is presented in this paper. Firstly, the knowledge in bonded repairs process was analyzed and summarized. Then, the composite repairs domain ontology framework was constructed and the classes with attributes, relationships and instances were defined. Finally, the relational database was used to realize the formal representation of the ontology. The experimental results show that the proposed method is effective.
\end{abstract}

Keywords- knowledge modeling; ontology; bonded repair; composite material

\section{INTRODUCTION}

Because of inherent features such as high strength, resistance to fatigue and lightweight and the mature digital design, manufacturing method, the application of composite aircraft component is more and more widely. The consumption of composite on the aircraft has become an advance mark. Along with design and manufacture of composite material structure, the bonded repair is born and its development direction oriented to high efficiency and reliability ${ }^{[1]}$. The damage repair process of composite component typically involving damage assessment and verification, maintenance design based on finite element analysis and computer simulation based maintenance process depends on the maintenance knowledge and experience. The related knowledge and experience determines the quality and efficiency directly. For maintenance enterprise, the knowledge in this process is the important intellectual resource and is the important embodiment of core competitiveness. With the development of the application of composite materials, a lot of maintenance cases have been accumulated in the field of aviation maintenance. In addition, the ultra manual repairs in maintenance practices rely on the existed similar cases which can provide the referenced experience and knowledge. Therefore, how to model this kind of knowledge to implement knowledge management, inheritance and reuse is very important. This can improve maintenance quality, shorten the repair cycle and achieve fast response of the maintenance. Therefore, this is one of the important ways to enhance the core competitiveness of related enterprises.

Nowadays, the competition between the countries and enterprises has been focused on knowledge competition. On the level of enterprises, how to manage and apply the accumulated and continuous absorbed knowledge has become a hot topic in industry and scientific research institutions. The knowledge modeling technologies based on a set of abstract model to generate easy to absorb, sharing and comprehensive application of knowledge representation that has thus become the most effective solution of this problem ${ }^{[2]}$. According to knowledge modeling in the field of composite material, Sapuan et al [3] reviewed the composite design based on concurrent engineering and the research status of material selection method and laminate design based on the knowledge were analyzed. Sun et al [4] proposed an algorithm to model process knowledge for injection molding process. The knowledge acquisition, representation and knowledge based process design framework in the process of injection molding of composite materials were studied by Liana et al [5]. Mei et al [6] developed a knowledge-based system for the design of aircraft composite component considering the characteristic of composite component design. This system was integrated with CATIA software. Fu et al [7] based on ASP.NET and SQL Server technology to realize the multi user cooperation, multi expert knowledge support of the composite expert design system. Liu et al [8] developed the connection design knowledge base based on B/S Structure. Wang et al [9] developed an expert system of composite laminate stacking sequence optimization which composed by the objective function library, local search operator library and ply rule library. Kim et al [10] established an expert system for the optimization design of composite laminate. The system integrates a general ply sequence optimization algorithm, the finite element analysis tool and the post-processing module. To support the engineering of laminated composite component, Premkumar et al [11] developed a novel semantic laminated composites knowledge management system which was based on a suit of ontologies for laminated composites materials and design for manufacturing and their integration.

The knowledge modeling methods related to composite components are almost involving the design and manufacturing process. The researches on structure repair of composite are less. This is not matched with the trend of widespread application of composite components in aircraft industry. This paper focuses on knowledge modeling for bonded repair of composite structures. Based on the process of repair, the 
knowledge classification and domain ontology were constructed to accumulate and apply knowledge for related enterprises. The rest of this paper is organized as follows: Section 2 describes the maintenance process of composite structures; Section 3 addresses in detail the knowledge modeling method; Section 4 shows original experimental knowledge modeling system and in Section 5, some conclusions and the future work are given.

\section{KNOWLEDGE BASED BONDED REPAIR OF COMPOSITE COMPONENT}

The primary objective of bonded composite repair technique is to restore the strength and stiffness of a damaged component and bring its original service condition back as much as possible. The bonded structural composite repair process is as follow: (1) the nondestructive detective technique is used to accurately locate the damage position and determine the size of damage zone. (2) The maintenance design which is considered as determining the bonded repair method according to the repair context. For little damage, the damage zone is injected by resin or resin and fiber mixture. For large damage, scarf or doubler based structural repair method are used. The geometry shape, material selection, adhesive selection, adhesive joints, laminates sequence, surface preparation, cure temperature and pressure et al are designed during this stage. (3) The structural maintenance craft is a key process parameter in bonded composite structural repairs. According to the results of the design, the damaged region needs to be accurately machined to remove material and taper edges for bonded repair. The surface preparation need execute in this stage and proper maintenance equipment with cure parameters need choose to complete the bonded composite repair. (4) Validation and Assessment steps are implemented by non destructive techniques. The bonded repaired structure need verify that its safety and integrity are restored.

\section{KNOWLEDGE MOdELING OF COMPOSITE STRUCTURAL REPAIR}

\section{A. Knowledge Analysis of Bonded Composite Structural Repair}

Bonded structural repair of composite components is a typical complex engineering problem, which need to consider many factors. The relationship between different factors may also be a contradiction or coupling. So the maintenance plan formulation of the composite structural repair is a knowledge intensive work which embodies core competence of the enterprise. Combined with the manual and previous research of bonded structural repair of composite, the knowledge of structural repair is mainly divided into the following several aspects.

(1) Type and geometrical features of the composite component. There are two typical types of composite components, which are laminated structure and sandwich structure. In recent years, the advanced composite material molding technology has produced a new structure, which is $3 \mathrm{~d}$ braided structure. And the composite laminated structure can be further subdivided into skin, wall panels, beams and ribs with typical geometrical features. The geometry of composite component (common geometric features including plane, quadratic surface and freeform surface, etc.) is the embodiment of its function as well as a key factor of maintenance design need to be addressed, such as the patch reflects the local geometry of the component, the finite element analysis depends on the geometry to establish accurate finite element model and the damaged material removal in the maintenance process need the geometry to generate cutter location file in the NC machining.

(2) Damage features of composite component, including damage type, damage reason, location and size of the damage zone, etc... There are typical damage types which are delaminating, debonding, core damage, perforation, moisture absorption, corrosion and matrix cracking etc. Common damage causes include impact, overload, extrusion, lightning, etc. There is a complex relationship between the cause of damage and damage types, that is, a kind of damage may cause multiple damage types, and a type of damage may be the result of multiple causes. The technicians depend on knowledge and experience to determine damage types and causes of damage. The location and size of damage area is used to maintenance design and process.

(3) Damage detection features of composite component, including detection methods, such as ultrasonic c scanning, $\mathrm{x}$ ray detection, shearography and eddy current testing etc. Damage detection results, such as damage image, audio, etc. Due to the anisotropy material behavior and overlapping molding, there is not any methods can inspect all types of damages. So the nondestructive detection method for a specific damage has an important reference value in the process of composite structural repair.

(4) Maintenance design. Maximum repair efficiency and quality and minimize the risk of structural failure is the objective of structural repair design. Using the patch repair the damage to recover the mechanical properties of the components, such as strength, stiffness etc need to accurately analysis and design the patch. The key content of maintenance design is selected proper maintenance parameters including geometry parameters, material parameters, ply design, adhesive selection, curing temperature and pressure etc. This process is a typical redesign process of composite component.

(5) Maintenance craft. The damage area needs to be removed accurately and generate the required geometry which is determined by maintenance design. This process involves material removal technique, process equipment, adhesive surface treatment method and equipment, curing parameters and equipment etc.

(6) Maintenance validation which is mainly used for evaluating airworthiness of the repaired composite component includes appearance inspection, internal quality test and performance test, etc. The inspection content is need to applied in the maintenance plan and provided the basis for regulatory requirements. 


\section{B. Composite Structural Repair Knowledge Ontology}

1) Ontology framework: The knowledge analysis of the bonded composite structural repair enumerated the professional terms and concepts which formed the vocabulary without organizational structure in this domain. The objective of the knowledge ontology construction is to classify them based on the logical rules of this domain, and then generate a knowledge framework structure clearly. Although the knowledge in the bonded composite structural repair domain has the nature of complexity, uncertainty and experience, but the composite component as a kind of typical aircraft structure has the characteristics of hierarchy. In addition, the composite component and its damage can be described by a set of physical property values. The maintenance process of each case has similar steps and obeys the same rules. This is the logical structure of the ontology framework.

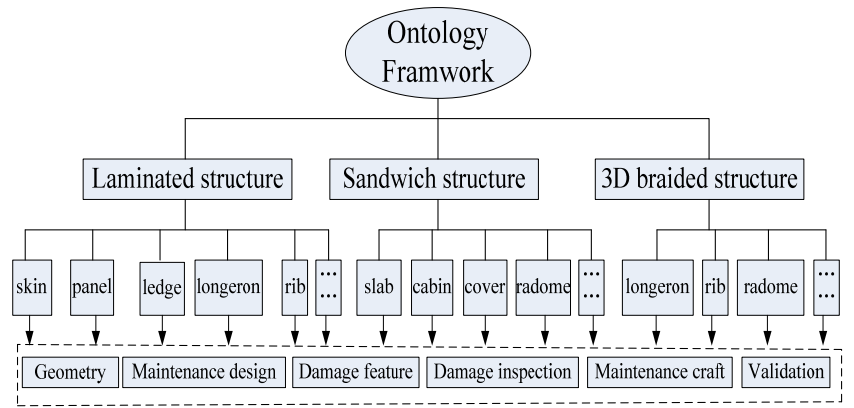

FIGURE I. THE KNOWLEDGE ONTOLOGY FRAMEWORK OF BONDED COMPOSITE STRUCTURAL REPAIR.

Figure 1 shows the ontology framework. The knowledge ontology is divided into three levels by this framework. The type of composite component covers the function and also produces a different geometry and laminate set etc. Each bonded composite structural repair contains six features such as component geometry, damage inspection, damage feature, and so on. According to each type structural repair, the object oriented knowledge model is established as shown in Figure 2.

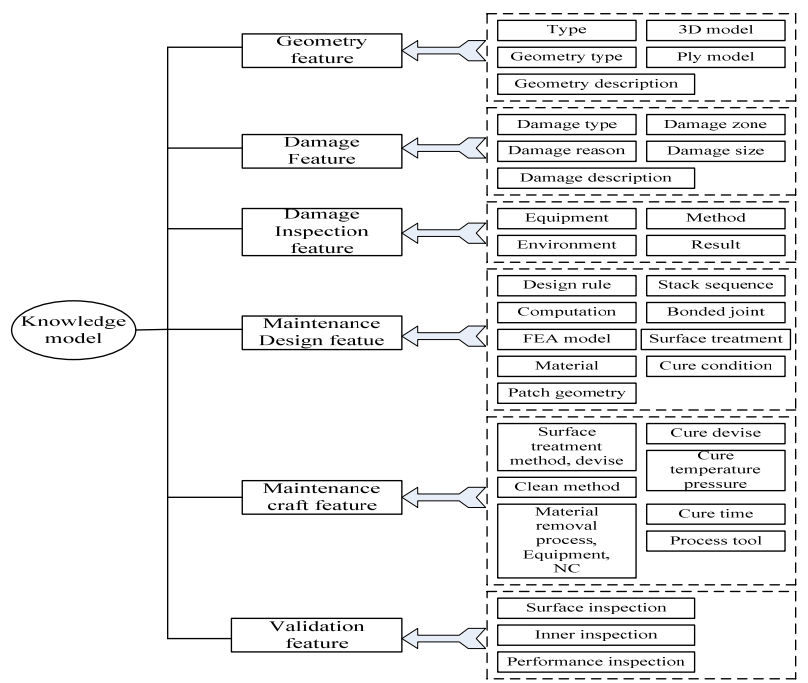

FIGURE II. THE OBJECT ORIENTED KNOWLEDGE MODEL.
2) Ontology element: The knowledge ontology consists of classes, relations, attributes and instances. The real object is described abstractly by the class. In this paper, the knowledge represented by text box in the ontology framework is an ontology class. The inheritance relationship between the classes of knowledge ontology forms of the tree structure. The "laminated structure", "sandwich structure", "3D braided structure" as the abstract class to be used for the induction of similar knowledge, was the basis of knowledge ontology construction and knowledge retrieval. These classes do not have the knowledge instances. The rest of the class as an instance class can have instance knowledge.

Relation is used to define the intrinsic connection between the classes. The relations in the knowledge ontology of bonded composite structural repair included inheritance, composition, aggregation and correlation. The relationship of inheritance reflects the connection between the parent and the child class. The child has all the attributes of the parent and is the extension of the parent class, such as the "skin" class is the child of the "laminated structure" class. The composition relation reflects the relationship between the parts and the whole, such as the composite component geometry feature class is part of the skin class. The aggregation relation is inverse relationship of the composition relation. The correlation has the characteristics of transitivity and symmetry, such as the relationship between the composite components geometrical feature class and the maintenance design class.

The attribute defined the property of the class. The most basic attribute is the class name. The attribute can be a numerical, text, CAD model, image, etc. In particular, a class can be used as attribute of another class, such as the maintenance craft class is the attribute of the skin class. In this paper, we only give the attributes of component geometry feature class: "ID, ParentID, Geometry type, Geometry description, 3D model, laminate, laminate set”.

The instance is the implementation of the class. According to the ontology framework, the classes' instances in second level corresponded to the maintenance cases. Based on the construction process of ontology elements, the knowledge ontology describes the domain knowledge clearly which formed the knowledge network. The instance describes the specific maintenance case, realizes the knowledge organization and representation which is easy for knowledge retrieval and reuse.

3) Ontology implementation: Domain ontology construction can be described by RDF, OIL, DAML, XML, OWL etc. Due to the big quantity of bonded composite structural repair knowledge and oriented to enterprise level application, the domain ontology needs higher efficiency and role management. This paper chooses relational database to realize the knowledge ontology. The abstract class table, instance class table, geometry feature table, damage feature table were constructed and the type of column with foreign key constrain relationship were employed to realize classes, relations and attributes of the knowledge ontology, as shown in Figure 3. 


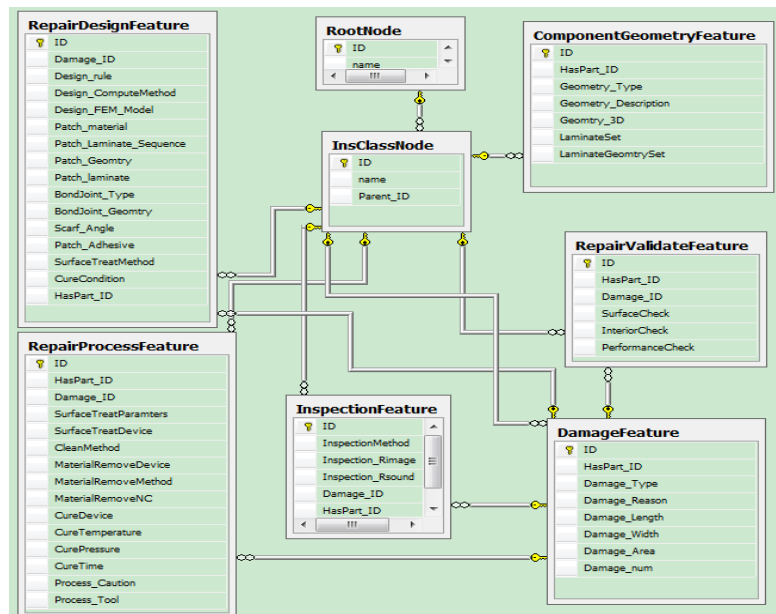

FIGURE III. THE KNOWLEDGE ONTOLOGY DESCRIPTION.

\section{IMPLEMENTATION AND ORIGINAL SYSTEM}

The proposed knowledge modeling method was written in VC\# on vs2008 development platform with SQLServer2005 relationship database. Figure 4 shows the prototype system interface. The tree structure in the object browser reflects the basic framework of the knowledge ontology. The six classes of the ontology such as geometry feature, damage feature of composite component are associated with knowledge instance by the command buttons. The command buttons are directly related to the corresponding knowledge instances in the database. The attribute window is used to modify and query the ontology framework. A typical case shows in the prototype system interface which describes the knowledge modeling result of the skin composite component clearly. The bonded structural composite repair knowledge modeling can finish by step by step mode in the prototype system and meanwhile finish the knowledge retrieval. The experimental result shows that the proposed method is effective. The proposed knowledge modeling method provides a solution for knowledge accumulation and application of related enterprises.

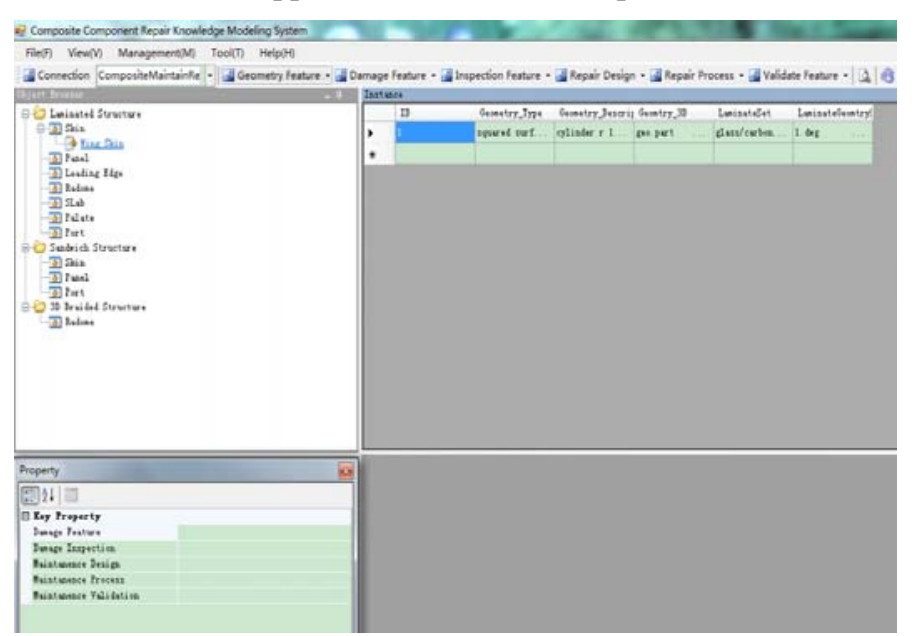

FIGURE IV. THE PROTOTYPE INTERFACE OF BONDED STRUCTURAL

\section{CONCLUSIONS}

With the application of composite materials in aircraft manufacturing industry, the bonded structural repair has become a new opportunity and challenge for aviation maintenance industry. The damage mechanisms in composites are often very complex because of their inherent nonhomogenous, anisotropic material behavior and lay-up molding. So the bonded composite structural repair is extremely dependent on the experience and accumulated knowledge. The accumulated composite repair knowledge as import intellectual resources of relevant enterprises is the embodiment of the core competitiveness in the current era of knowledge economy. The knowledge modeling method presented in this paper provides an effective solution for aircraft maintenance enterprise for knowledge accumulation and application. The stability and reliability of bonded structural composite repair technique is also promoted. At the same time, the method can also be effective in improving the efficiency which has widespread application prospect in engineering domain.

\section{ACKNOWLEDGMENT}

This work was supported by the National Natural Science Foundation Civil Aviation Joint Fund key project of China under Grant No. U1233202 and General Project of Civil Aviation Flight University of China under Grant No. J2014-37.

\section{REFERENCES}

[1] Katnam K, Silva D, Young T. Bonded repair of composite aircraft structures: A review of scientific challenges and opportunities [J]. Progress in Aerospace Sciences, vol 61, pp26-42,2013.

[2] Chandrasegaran S K, Ramani K, Sriram R D, et al. The evolution, challenges, and future of knowledge representation in product design systems [J]. Computer-Aided Design, 2013, vol 45, pp204-228.

[3] Sapuan S M, Mansor M R. Concurrent engineering approach in the development of composite products: a review [J]. Materials \& Design, 2014, vol 58, pp161-167.

[4] Sun W, Lin F, Hu X. Computer-aided design and modeling of composite unit cells [J]. Journal of Composite Science and Technology, 2001, vol 61, pp289-299.

[5] Liana M. A framework for knowledge acquisition, representation and problem- solving in knowledge-based planning [D]. Michigan State University, 2001

[6] Mei Zhongyi, Zhu Sanshan, Younus Muhammad, et al. Research on knowledge-based system for typical aircraft composite component design [J]. Procedia Engineering, 2011, vol 15, pp1431- 1435.

[7] Fu Wei, Wang Peng, Li Qingfen. Study on expert system in composite material design based on the web $[\mathrm{J}]$. Journal of Harbin Engineering University, 2004, vol 25, pp773-776.

[8] Liu Zhicun, Xiao Xinghui. Researching knowledge based system for aircraft manufacturing composite material part connection design [J]. Manufacturing Automation, 2013, vol 35, pp154-156.

[9] Wang Gongdong, Wang Jun, Chen Hao, et al. Development of an expert system for composite laminate stacking sequence optimization [J]. Journal of Wuhan University of Technology, 2013, vol 35, pp43-49.

[10] Kim J. Development of user friendly expert system for composite laminate design [J].Composite Structures, 2007, vol 79, pp76-83.

[11] Premkumar V, Krishnamurty S, Wileden J C, et al. A semantic knowledge management system for laminated composites [J]. Advanced Engineering Informatics, 2014, vol 28, pp91-101. 